\title{
Neutrophil Lymphocyte Ratio as an Inflammatory Marker in Chronic Kidney Disease: Determinants and Correlates
}

\section{Peter Kehinde Uduagbamen ${ }^{1 *}$, Adesola Temitope Oyelese ${ }^{2}$, Abdallah Olukayode AdebolaYusuf ${ }^{3}$, Mary Umoh Thompson ${ }^{3}$, Boladale Ajani Afeez Alalade ${ }^{4}$, Osaze Ehioghae ${ }^{1}$}

\author{
${ }^{1}$ Division of Nephrology and Hypertension, Department of Internal Medicine, Ben Carson (Snr) School of Medicine, Babcock \\ University/Babcock University Teaching Hospital, Ilishan-Remo, Nigeria \\ ${ }^{2}$ Department of Haematology and Blood Transfusion, Ben Carson (Snr) School of Medicine, Babcock University/Babcock \\ University Teaching Hospital, Ilishan-Remo, Nigeria \\ ${ }^{3}$ Division of Radiodiagnosis, Department of Surgery, Ben Carson (Snr) School of Medicine, Babcock University/Babcock \\ University Teaching Hospital, Ilishan-Remo, Nigeria \\ ${ }^{4}$ Endocrine, Diabetes and Metabolism Unit, Department of Internal Medicine, Federal Medical Centre, Abeokuta, Nigeria \\ Email: * petr.uduagbamen@gmail.com
}

How to cite this paper: Uduagbamen, P.K., Oyelese, A.T., AdebolaYusuf, A.O., Thompson, M.U., Alalade, B.A.A. and Ehioghae, O. (2022) Neutrophil Lymphocyte Ratio as an Inflammatory Marker in Chronic Kidney Disease: Determinants and Correlates. Open Journal of Nephrology, 12, 23-35. https://doi.org/10.4236/ojneph.2022.121003

Received: December 2, 2021

Accepted: January 15, 2022

Published: January 18, 2022

Copyright $\odot 2022$ by author(s) and Scientific Research Publishing Inc. This work is licensed under the Creative Commons Attribution International License (CC BY 4.0)

http://creativecommons.org/licenses/by/4.0/

(c) (i) Open Access

\begin{abstract}
Introduction: Inflammation has been implicated as a major reason for the higher morbidity and mortality in chronic kidney disease (CKD) compared to the diseases that commonly precedes it. The neutrophil lymphocyte ratio (NLR) has increasingly been reported to be a marker of systemic inflammation. We studied the neutrophil lymphocyte ratio and its relationship with kidney function and other markers of inflammation in health and in CKD. Methods: Two hundred and forty four participants in three cohorts: healthy, CKD stage 1 - 2 and, stage 3 - 4, were studied. Data of clinical, NLR, uric acid, urine albumin creatinine ratio (UACR), electrolytes were documented and independent associates of NLR were determined. Results: The NLR was higher in the CKD cohorts, $\mathrm{P}<0.001$ and females, $\mathrm{P}=0.01$. The mean NLR of all participants, the healthy and, CKD cohorts were $2.8 \pm 0.7,1.5 \pm 0.6$ and $3.9 \pm 1.4, \mathrm{P}<0.001$. The mean NLR of all participants $\geq 65$ years, all males $\geq$ 65 years and, all females $\geq 65$ years were $4.0 \pm 1.6,3.7 \pm 1.0$ and $4.2 \pm 1.2, \mathrm{P}=$ 0.01 . The NLR was positively related to the age $(\mathrm{P}<0.001)$, systolic blood pressure $(\mathrm{P}=0.012)$, uric acid $(\mathrm{P}=0.018)$, UACR $(\mathrm{P}=0.006)$ and platelet lymphocyte ratio, $\mathrm{P}=0.04$. The NLR was negatively related to the hematocrit $(\mathrm{P}<0.001)$, albumin $(\mathrm{P}<0.001)$ and glomerular filtration rate $(\mathrm{P}<0.001)$. Multivariate analysis after ruling out cofounders, showed age (aOR5.8, CI4.26 - 10.22), systolic hypertension (aOR1.5, CI-1.21 - 2.07), hyperuricemia (aOR-1.5, CI-0.94 - 2.09), elevated urine ACR (aOR-1.7, CI-1.25 - 2.47) and
\end{abstract}


CKD (aOR-7.2, CI-1.45 - 8.94) as independent predictors of NLR. Conclusion: The NLR as an inflammatory marker is elevated in chronic kidney disease, and increases with disease severity hence it can be a useful tool in determining the presence and severity of inflammation in CKD.

\section{Keywords}

Neutrophil Lymphocyte Ratio, Inflammation, Chronic Kidney Disease, Platelet Lymphocyte Ratio, Hyperuricemia, Albuminuria

\section{Introduction}

Chronic kidney disease (CKD) has continued to pose great health challenges to its victims worldwide despite vast literature on its pathophysiologic mechanisms [1]. Mortality from the disease has persistently gone beyond the sequel of traditional cardiovascular risk factors which could also be classified as pre CKD conditions particularly, diabetes, hypertension, dyslipidemia and obstructive uropathy among others [2]. Though the pre CKD conditions are mostly inflammatory, the increased mortality in CKD compared to any of these conditions has been attributed to more tissue destruction secondary to the persistent inflammatory cascade seen in CKD.

The neutrophil lymphocyte ratio (NLR) has long been identified as an inflammatory marker whose level can predict the level of severity of inflammatory conditions such as CKD [3]. The high prevalence of cardiovascular disease in $\mathrm{CKD}$ has been reported to be associated with higher levels of inflammatory makers and this has subsequently been implicated as a contributor to the poor adverse outcome of CKD compared to pre CKD conditions [2]. Markers of inflammation could appear in blood, urine or feces or other body fluids before, during or after the onset of symptoms of disease. While markers of systemic inflammation are mostly elevated in diseases, others could be depressed. Commonly used inflammatory markers in supporting diagnosis, monitoring progression/remission or prognostication include: IL-1, IL-6, fibrinogen, plasma viscosity, ferritin, tissue necrosis factor (TNF), erythrocyte sedimentation rate (ESR) and C-reactive protein (CRP) [2] [3].

The commonly seen cardiovascular and central nervous (CNS) disorders in CKD have been attributed to the pan systemic inflammation in CKD [4]. The inflammation-induced tissue remodeling precedes, and continues with CKD and could progress to tissue fibrosis and loss of renal functional capacity leading to proteinuria, atherosclerosis, left ventricular hypertrophy (LVH), cerebral atrophy and textural changes in the brain parenchyma [5] [6]. Proteinuria and LVH have been associated with increased cardiovascular events and mortality in CKD [5]. The hyperuricemia commonly seen in CKD is also reported to augment the inflammatory cascade in CKD due to the endothelial tissue injury, a pivotal oc- 
currence in the filtration apparatus of the kidneys [7].

Higher neutrophil counts are commonly seen in inflammatory, infective and stressful states but lower platelets and lymphocytes are often associated with general poor health particularly chronic conditions [8]. Elevated NLR has been reported to predict mortality and heart failure in patients with coronary artery disease, as it predicts CKD occurrence in hypertension and mortality in some cancers [9], in addition to predicting poor prognostic outcome in acute coronary syndromes, particularly, with ST elevation [10].

Despite the volume of literature on NLR as an inflammatory marker, its associations with other known markers of inflammation in CKD such as uric acid and albuminuria have not been completely elucidated coupled with an almost absent literature on NLR and its correlates in sub Sahara Africa. We studied NLR as an inflammatory marker in CKD and determined its correlation with serum uric acid and albuminuria in a low income setting.

\section{Materials and Methods}

This was a hospital based descriptive study in which patients 16 years and older, with CKD according to the $2012 \mathrm{KDOQI}$ criteria [11] and receiving treatment at the nephrology and hypertension clinic of Babcock University Teaching Hospital, Ilishan-Remo between January and July 2021 were consecutively recruited after giving informed consent. The healthy volunteers were recruited from the staff and patients who had previously been treated for acute disease conditions at the Family Medicine clinics of Federal Medical Centre, Abeokuta. The participants were grouped into three: healthy controls, CKD stages $1-2$ and, stage 3.4. All patients had renal ultrasound scan (RUS) where obstructive lesions and secondary causes of hypertension and kidney disease were rule out by determining the flow pattern (using Doppler) and the resistivity indexes.

Exclusion criteria included patients with any acute disease, infections, cancers, connective tissue diseases, diabetes, heart failure, chronic liver disease (CLD), blood dyscrasias and other hematologic disorders, frequent users of non-steroidal anti-inflammatory drugs (NSAIDs) and those who were using or who at the time of recruitment, had used within the preceding 6 months, steroids and/or heavy metal containing soaps, creams, ointments or "eye paints".

All participants gave detailed history covering socio-demographics, family and personal illnesses, drug use (prescribed and non-prescribed) and their hospital case files were retrieved to rule out any of the exclusion criteria. The height and weight were measured using standardized protocols and the BMI was calculated. The blood pressure was taken in the sitting position after five minutes rest, with the back and arm resting on a support.

All participants were tested for microalbuminuria using the Micra Albustix Test strip to determine the urine albumin creatinine ratio (UACR). The premenopausal women were asked to abstain from urine collection a day to, and up to a day after, their menstrual flow. All females were asked to clean the vulva and 
part the labia to collect urine. The micra albustix bottle was closed immediately after taking a strip out. The end of the strip with pad was completely immersed into the urine to cover the full length of the pads for 50 seconds and then remove by rolling it against the edge of the universal bottle to remove excess urine. The strip pad color was matched with the "strip pad color" inscribed on the strip container and the results were documented.

Venous blood sample was collected from a peripheral vein in the sitting position, in room air for analysis of the full blood count (FBC), erythrocyte sedimentation rate (ESR) and, the serum electrolytes, urea, creatinine and uric acid. The creatinine-based glomerular filtration rate (GFR) was calculated using the CKD epidemiological collaboration (CKD-EPI) formula [12].

\section{Definitions}

Diabetes: diagnosis or drug treatment of diabetes or a fasting blood glucose (FBG) of $\geq 7.0 \mathrm{mmol}$ [13].

Hypertension: elevated blood pressure (BP) $\geq 140 / 90 \mathrm{mmHg}$ [14].

Microalbuminuria: ACR $>3.4 \mathrm{mg} / \mathrm{mmol}$ [15].

Hyperuricemia: uric acid (UA) $>0.42 \mathrm{mmol} / \mathrm{l}$ (males); $0.36 \mathrm{mmol} / \mathrm{l}$ (females) [16].

Anemia: hematocrit $<39 \%$ (males) and $<36 \%$ (females) [17].

Elevated NLR: $>3$ [18].

Elevated PLR: $>160$ [18].

Metabolic acid: bicarbonate $<22 \mathrm{mmol} / \mathrm{l}$ [19].

CKD: stage 1 and 2 (GFR $\geq 60$ ); stages 3 - 4 (GFR 15.0 - 59.9) [12].

Statistical analysis

Continuous variables were presented as mean with standard deviation and compared using student's t-test while categorical variables were presented as proportions with frequencies and compared using Chi-square. Correlation was done by linear regression analysis using Spearman's correlation. Variables with a $\mathrm{p}$ value of $<0.25$ on univariate analysis were entered into the multivariate model, with backward elimination to adjust for confounders, to determine independent predictors of elevated NLR [20]. The p-value $<0.05$ was considered statistically significant.

The research followed the tents of the Declaration of Helsinki. The study was approved by the Babcock University Human Research Ethics Committee (BUHREC) and the Human Ethics Committee of the Federal Medical Centre.

\section{Results}

A total of two hundred and forty four (127 men and 117 women) participants were studied. The participants were grouped into 3: those in health, CKD stages 1 and 2 and, CKD stages 3 and 4 (Table 1). The mean age of all participants, males and females were $49.9 \pm 7.3 \mathrm{yrs}, 49.2 \pm 6.8 \mathrm{yrs}$ and $50.7 \pm 8.2 \mathrm{yrs}, \mathrm{P}=0.07$. A greater proportion of participants 65 yrs and older had CKD stage 3 or 4 . The 
Table 1. Sociodemographic and clinical characteristics of participants.

\begin{tabular}{cccccc}
\hline Variables & Total & Healthy & CKD 1 - & CKD 3 - 4 & ANOVA \\
\hline & N $=244(\%)$ & $\mathrm{N}=100(\%)$ & $\mathrm{N}=76(\%)$ & $\mathrm{N}-68(\%)$ & \\
\hline Mean $\pm \mathrm{SD}$ & Mean $\pm \mathrm{SD}$ & Mean $\pm \mathrm{SD}$ & Mean $\pm \mathrm{SD}$ & \\
\hline Sex, & & & & & \\
Males & $127(52.0)$ & $45(45.0)$ & $44(57.9)$ & $38(55.9)$ & 0.04 \\
Females & $117(48.0)$ & $55(55.0)$ & $32(42.1)$ & $30(44.1)$ & \\
Age, (mean) years & $49.9 \pm 7.3$ & $46.0 \pm 4.2$ & $51.5 \pm 8.6$ & $53.9 \pm 10.4$ & 0.001 \\
$16-39$ & $78(32.0)$ & $37(37.0)$ & $29(38.2)$ & $12(17.7)$ & 0.001 \\
$40-64$ & $131(53.7)$ & $49(49.0)$ & $39(51.3)$ & $43(63.2)$ & \\
$\geq 65$ & $35(14.3)$ & $14(14.0)$ & $8(10.5)$ & $13(19.1)$ & \\
\hline Smoking & & & & & \\
Yes & $13(5.3)$ & $11(11.0)$ & $2(2.6)$ & $0(0.0)$ & $<0.001^{*}$ \\
No & $231(94.7)$ & $89(89.0)$ & $74(97.4)$ & $68(100.0)$ & \\
Mean BMI, kg/m ${ }^{2}$ & $26.1 \pm 5.9$ & $25.6 \pm 6.1$ & $25.3 \pm 4.5$ & $27.9 \pm 5.7$ & 0.07 \\
Mean SBP, mmHg & $125.7 \pm 7.0$ & $117.6 \pm 6.2$ & $122.5 \pm 8.8$ & $141.3 \pm 8.9$ & $<0.001$ \\
Mean DBP, mmHg & $77.3 \pm 6.3$ & $72.4 \pm 4.7$ & $75.9 \pm 6.3$ & $86.1 \pm 7.7$ & 0.001 \\
\hline
\end{tabular}

CKD-chronic kidney disease, ${ }^{*}$-Fisher's exact test, BMI-body mass index, SBP-systolic blood pressure, DBP-diastolic blood pressure.

BMI and the blood pressures of the CKD cohorts were higher than the healthy participants.

The serum bicarbonate concentration (SBC), hematocrit, albumin and lymphocytes were lower in the CKD 3 - 4 cohorts compared to the CKD 1 - 2 and, the healthy cohorts, $\mathrm{P}=0.03,0.001, \mathrm{P}=0.002$ and $\mathrm{P}=0.002$ (Table 2). The mean NLR of all participants, the healthy and, CKD cohorts were $2.8 \pm 0.7,1.5 \pm$ 0.6 and $3.9 \pm 1.4, \mathrm{P}<0.001$. The mean NLR of all participants $\geq 65$ years, all males $\geq 65$ years and, all females $\geq 65$ years were $4.0 \pm 1.6,3.7 \pm 1.0$ and $4.2 \pm$ $1.2, \mathrm{P}=0.01$. The serum creatinine, uric acid, urine ACR, neutrophil, NLR and PLR were higher in the CKD 3-4 cohorts compared to the CKD $1-2$ and, the healthy cohorts, $\mathrm{P}<0.001, \mathrm{P}<0.001, \mathrm{P}<0.001, \mathrm{P}=0.001, \mathrm{P}<0.001$ and $\mathrm{P}=0.04$ respectively.

Elevated NLR was associated with the female gender $(\mathrm{P} 0.04)$, aging $(\mathrm{P}<0.001)$ and systolic hypertension, $\mathrm{P}=0.012$ (Table 3 ), as it was associated with anemia $(\mathrm{P}<0.001)$, hypoalbuminemia $(\mathrm{P}<0.001)$, hyperuricemia $(\mathrm{P}=0.018)$, microalbuminuria $(\mathrm{P}=0.006), \mathrm{CKD}(\mathrm{P}<0.001)$ and elevated PLR $(\mathrm{P}=0.04)$.

Pearson correlation coefficient (Table 4) showed an insignificant positive association between NLR and the urine ACR. There was a weak but significant negative correlation between the NLR and GFR (Figure 1) and there was a very weak (insignificant) positive correlation between NLR and the uric acid (Figure 2). 
Table 2. Laboratory characteristics of participants.

\begin{tabular}{|c|c|c|c|c|c|}
\hline Variables & Total & Healthy & CKD 1 - 2 & CKD $3-4$ & ANOVA \\
\hline & $\mathrm{N}=244(\%)$ & $\mathrm{N}=100(\%)$ & $\mathrm{N}=76(\%)$ & N-68 (\%) & \\
\hline & Mean \pm SD & Mean \pm SD & Mean \pm SD & Mean \pm SD & \\
\hline & Median (R) & Median (R) & Median (R) & Median (R) & \\
\hline Bicarbonate, mmol/l & $21.7 \pm 5.3$ & $23.6 \pm 7.6$ & $21.2 \pm 4.4$ & $19.5 \pm 4.6$ & 0.03 \\
\hline Urea, mmol/l & $10.1 \pm 3.7$ & $7.1 \pm 5.5$ & $9.9 \pm 6.3$ & $14.6 \pm 6.9$ & 0.001 \\
\hline Creatinine, umol/l & $154.5 \pm 7.3$ & $83.8 \pm 6.6$ & $154.9 \pm 8.2$ & $259.5 \pm 9.5$ & $<0.001$ \\
\hline Uric acid, mmol/l & $459.7 \pm 12.3$ & $354.2 \pm 11.3$ & $497.4 \pm 11.8$ & $572.6 \pm 14.8$ & $<0.001$ \\
\hline $\mathrm{ACR}, \mathrm{mg} / \mathrm{g}$ & $29.1 \pm 6.1$ & $19.6 \pm 3.8$ & $31.2 \pm 5.4$ & $40.7 \pm 8.8$ & $<0.001$ \\
\hline Hematocrit, \% & $36.5 \pm 6.8$ & $41.5 \pm 7.3$ & $35.8 \pm 5.1$ & $29.9 \pm 3.2$ & 0.001 \\
\hline Albumin, mg/dL & $38.8 \pm 8.2$ & $42.3 \pm 8.8$ & $37.8 \pm 7.4$ & $34.7 \pm 7.1$ & 0.002 \\
\hline Low HDL (n, \%) & $89(36.5)$ & $19(19.0)$ & $29(38.2)$ & $41(60.3)$ & 0.002 \\
\hline Elevated LDL, (n, \%) & $86(35.2)$ & $21(21.0)$ & $34(44.7)$ & $31(45.6)$ & 0.06 \\
\hline Triglyceride, (n, \%) & $99(40.6)$ & $34(34.0)$ & $30(39.5)$ & $35(51.5)$ & 0.05 \\
\hline \multicolumn{6}{|l|}{ Leucocytes $10^{3} / / \mathrm{ul}$} \\
\hline median (range) & $5.3(3.1-7.8)$ & $5.4(3.8-6.2)$ & $5.8(3.8-6.9)$ & $6.1(4.9-7.8)$ & 0.05 \\
\hline \multicolumn{6}{|l|}{ Neutrophil $10^{3} / / \mathrm{ul}$} \\
\hline median (range) & $5.1(2.9-7.7)$ & $3.8(3.3-4.7)$ & $4.8(4.1-5.8)$ & $6.8(4.1-7.7)$ & 0.001 \\
\hline \multicolumn{6}{|l|}{ Lymphocytes $10^{3} / / \mathrm{ul}$} \\
\hline median (range) & $2.1(1.0-4.1)$ & $2.2(1.9-4.1)$. & $1.8(1.3-2.6)$ & $1.3(1.0-1.9)$ & 0.002 \\
\hline Platelets $10^{3} / / \mathrm{ul}$ & 205.3 & 228.4 & 195.2 & 183.2 & 0.04 \\
\hline (range) & $(155-289)$ & $(190-289)$ & $(168-226)$ & $(155-223)$ & \\
\hline NLR, median (range) & $2.8(0.9-7.2)$ & $1.5(0.9-2.1)$ & $2.3(1.7-3.4)$ & $5.3(2.6-7.2)$ & $<0.001$ \\
\hline PLR, median & 104.1 & 108.4 & 110.3 & 124.4 & 0.04 \\
\hline (range) & $(74.2-205.3)$ & $(74.2-197.3)$ & $(92.7-220.5)$ & $(104.3-205.3)$ & \\
\hline
\end{tabular}

CKD-chronic kidney disease, R-range, ACR-albumin creatinine ratio, NLR-neutrophil lymphocyte ratio, PLR-platelet lymphocyte ratio.

Table 3. Correlates of neutrophil lymphocyte ratio in participants.

\begin{tabular}{cccccc}
\hline Variables & NLR $<3.0$ & NLR $\geq 3.0$ & OR & 95\% CI & P-value \\
\hline Sex & & & & \\
Males & $105(82.7)$ & $22(17.3)$ & 1.44 & $1.03-1.96$ & 0.04 \\
Females & $91(77.8)$ & $26(22.2)$ & & & \\
Age, years & & & & \\
$<65$ & $163(85.8)$ & $27(14.2)$ & 5.1 & $1.38-6.46$ & $<0.001$ \\
$\geq 65$ & $33(61.1)$ & $21(38.9)$ & & & \\
\hline
\end{tabular}




\section{Continued}

\begin{tabular}{|c|c|c|c|c|c|}
\hline \multicolumn{6}{|l|}{$\mathrm{BMI}, \mathrm{kg} / \mathrm{m}^{2}$} \\
\hline$<25.0$ & $71(85.5)$ & $12(14.5)$ & 1.87 & $0.93-2.02$ & 0.030 \\
\hline$\geq 25.0$ & $125(77.6)$ & $36(22.4)$ & & & \\
\hline \multicolumn{6}{|l|}{$\mathrm{SBP}, \mathrm{mmHg}$} \\
\hline$<140$ & $148(85.1)$ & $26(14.9)$ & 2.77 & $1.89-3.97$ & 0.012 \\
\hline$\geq 140$ & $48(68.6)$ & $22(31.4)$ & & & \\
\hline \multicolumn{6}{|l|}{ Hematocrit, \% } \\
\hline$<39(\mathrm{M}) ;<36(\mathrm{~F})$ & $44(52.4)$ & $40(47.6)$ & 7.43 & $5.03-11.48$ & $<0.001$ \\
\hline$\geq 39(\mathrm{M}) ; \geq 36(\mathrm{~F})$ & $152(95.0)$ & $8(5.0)$ & & & \\
\hline \multicolumn{6}{|l|}{ Albumin, mg/dL } \\
\hline$<35$ & $54(60.0)$ & $36(40.0)$ & 5.92 & $5.24-10.16$ & $<0.001$ \\
\hline$>35$ & $142(92.2)$ & $12(7.8)$ & & & \\
\hline \multicolumn{6}{|l|}{ Uric acid, mmol/l } \\
\hline$<0.42(\mathrm{M}) ;<0.36(\mathrm{~F})$ & $92(91.1)$ & $9(8.9)$ & 2.83 & $2.05-4.18$ & 0.018 \\
\hline$\geq 0.42(\mathrm{M}) ; \geq 0.36(\mathrm{~F})$ & $106(73.1)$ & $39(26.9)$ & & & \\
\hline \multicolumn{6}{|l|}{$\mathrm{ACR}, \mathrm{mg} / \mathrm{mmol}$} \\
\hline$<3.4$ & $128(88.9)$ & $16(11.1)$ & 3.0 & $1.66-4.13$ & 0.006 \\
\hline$\geq 3.4$ & $68(68.0)$ & $32(32.0)$ & & & \\
\hline \multicolumn{6}{|l|}{$\mathrm{eGFR}, \mathrm{ml} / \mathrm{min}$} \\
\hline$<60$ & $30(44.1)$ & $38(55.9)$ & 7.9 & $5.29-12.59$ & $<0.001$ \\
\hline$\geq 60$ & $166(94.3)$ & $10(5.7)$ & & & \\
\hline \multicolumn{6}{|l|}{ PLR } \\
\hline$<160$ & $148(82.2)$ & $32(17.8)$ & 1.60 & $0.82-1.97$ & 0.04 \\
\hline$\geq 160$ & $48(75.0)$ & $16(25.0)$ & & & \\
\hline
\end{tabular}

OR-odds ratio, CI-95\% confidence interval, BMI-body mass index, SBP-systolic blood pressure, ACR-albumin creatinine ratio, eGFR-estimated glomerular filtration rate, PLRplatelet lymphocyte ratio.

Table 4. Pearson's linear correlation coefficient between NLR and eGFR, Uric acid and, ACR.

\begin{tabular}{ccccc}
\hline Variables & $\mathrm{r}$ & $95 \%$ CI & $\mathrm{P}$ & Correlation \\
\hline NLR and GFR of all participants & -0.144 & $(0.082-0.173)$ & 0.049 & weak negative significance \\
NLR and UA of all participants & 0.022 & $(0.020-0.031)$ & 0.764 & very weakly positive \\
NLR and ACR of all participants & 0.095 & $(0.093-0.101)$ & 0.194 & weakly positive \\
\hline
\end{tabular}

NLR-neutrophil lymphocyte ratio, eGFR-estimated glomerular filtration rate, ACR-albumin creatinine ratio, UA-uric acid.

Multivariate regression analysis (Table 5) showed that age (aOR5.8, CI-4.26 10.22), systolic hypertension (aOR1.5, CI-1.21 - 2.07), anemia (aOR-5.5, CI-3.59), hypoalbuminemia (aOR-4.9, CI-2.73 - 7.11), hyperuricemia (aOR-1.5, CI-0.94 2.09), elevated urine ACR (aOR-1.7, CI-1.25 - 2.47) and CKD (aOR-7.2, CI-1.45 - 8.94) as independent associates of high NLR. 


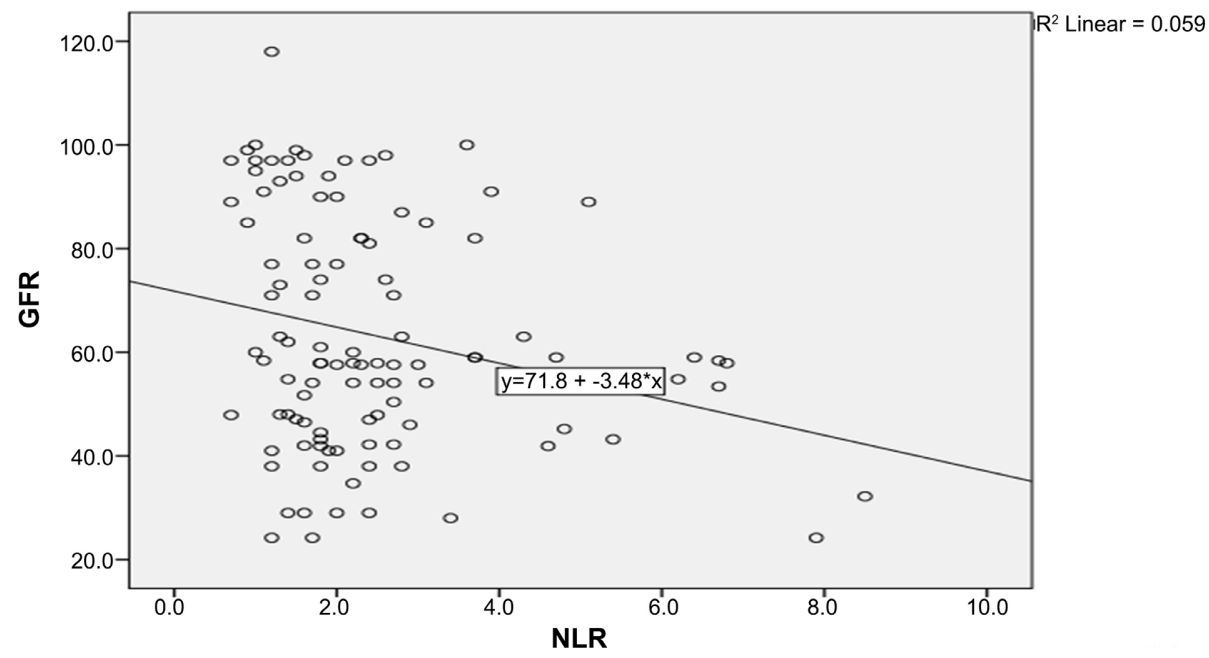

Figure 1. Pearson Correlation between NLR and eGFR. Very weakly significant negative correlation, $\mathrm{r}=-0.144, \mathrm{P}-0.049$.

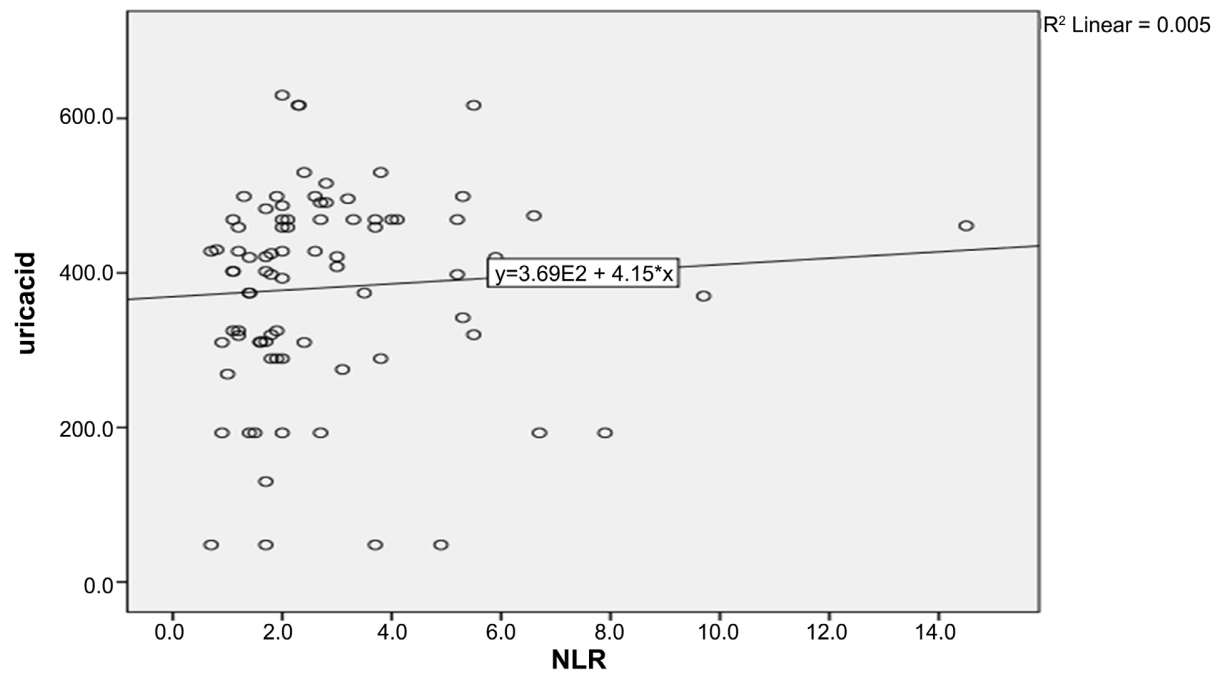

Figure 2. Pearson Correlation between NLR and Uric acid. Insignificant positive correlation: $\mathrm{r}=-0.022, \mathrm{P}-0.764$.

Table 5. Multivariate analysis showing independent associates of neutrophil lymphocyte ratio.

\begin{tabular}{cccc}
\hline Variables & aOR & $95 \%$ CI & P-value \\
\hline Age & 5.8 & $4.26-10.22$ & $<0.001$ \\
Systolic blood pressure & 1.5 & $1.21-2.07$ & 0.04 \\
Hematocrit & 5.5 & $3.59-9.13$ & $<0.001$ \\
Albumin & 4.9 & $2.73-7.11$ & $<0.001$ \\
Uric acid & 1.5 & $0.94-2.09$ & 0.03 \\
Urine albumin creatinine ratio & 1.7 & $1.25-2.47$ & 0.03 \\
Glomerular filtration rate & 7.2 & $1.45-8.94$ & $<0.001$ \\
\hline
\end{tabular}

aOR-adjusted odds ratio, CI-95\% confidence interval. 


\section{Discussion}

We assessed in our series the neutrophil lymphocyte ratio (NLR) as an inflammatory marker in CKD and its associations with other known inflammatory markers in CKD. To the best of our knowledge, our study is the first in sub Sahara Africa (SSA) to assess this association. We found a significant increase in NLR from health through CKD stage 1 and 2 to stage 3 and 4. The NLR was positively correlated with markers of inflammation: uric acid, urine albumin creatinine ratio (UACR) and the platelet lymphocyte ratio (PLR), as it was negatively related to the glomerular filtration rate, hematocrit and albumin. Hypertension (systolic) and obesity, both associated with increased inflammatory risk, were positively correlated with the NLR. De Cuiceis et al. [21] had reported an association between NLR and the inflammatory changes in the vasculature that could progress to atherosclerosis, hypertension and cardiovascular disease and events. Obesity is a known associate of inflammatory changes associated with lipid peroxidation, our finding of a positive relation between obesity and NLR further strengthen NLR as an inflammatory marker in CKD [22].

In inflammatory states of whatever source including stress, the involvement of the immune system mediates the recruitment of leucocytes, particularly neutrophils, mostly with a concurrent decrease in the lymphocytes during chronic illness associated with poor QOL and increased morbidity [23]. The positive association between NLR and hyperuricemia in this study also strengthen NLR as an inflammatory marker. Hyperuricemia induces an anti-oxidative state in the extracellular space, however, in the intracellular and intravascular compartments, along with other mediators of chronic inflammatory states, it induces renal microvascular/endothelial injury associated with release of vasogenic cytokines, decrease release of endothelium-derived nitric oxide (eNO) and prostaglandins [24]. The chronic vasoconstriction in the vascular bed favors the laying down of fibro-fatty deposits that progresses to atherosclerosis, associated with left ventricular hypertrophy with increased tendency for non-dipping blood pressure, a known risk factor for cardiovascular events and death [25]. The increased activity of sodium transporters in the renal proximal tubules secondary to hyperuricemia only worsens the hypertension and the cardiovascular diseases associated with hyperuricemia [26]. The increased production of the more atherogenic units of the low density lipoproteins (LDL) secondary to excessive renal protein (albumin) loss further aggravate the dyslipidemia that is common in CKD [27]. Though we didn't assess the relationship between NLR and cardiovascular disease and events, its positive association with systolic hypertension in the CKD cohorts, suggest an increased likelihood of cardiovascular disease and events in the future as the inflammatory cascade often runs a chronic course [28].

Chronic immunologic injury involving the renal bed leads to alteration of the lining of protein-like ligands on the endothelial surfaces as they undergo surface-capping thereby shedding immune complexes into the subepithelial spaces [29]. The foot processes of the glomerular visceral membrane are progressively 
replaced by continuous cytoplasmic bands along the glomerular basement membrane losing their charge and size selectivity. The resulting podocyte "fusion" or "effacement" causes the loss of albumin and other large and/or negatively charged substances into the urine. The protein load on the renal filtration barrier induces an inflammatory injurious state causing a back flow of glomerular ultrafiltrate worsening the proteinuria. Microalbuminuria is a known inducer and facilitator of progressive kidney damage, and an initiator and facilitator of cardiovascular disease and events [30].

The higher NLR in the elderly in our study mirrors previous findings [21] [22]. The aging process is associated with several comorbidities associated with debilitating diseases, that cause suppressed lymphocyte release and activation. The higher NLR in women in our study is not in agreement with findings by Zang et al. [31]. We infer that the higher proportion of females among the elderly coupled with the loss of the hitherto protective estrogen in the post-menopausal year induces the reported higher inflammatory and cardiovascular risk in them compared to men of similar age. We found a declining pattern of platelets and lymphocytes in the CKD cohorts compared to the healthy cohorts. A significantly rising ratio as we found could be attributed to a greater decrease in the lymphocyte count as CKD progresses.

The negative relationship between NLR and the serum bicarbonate, hematocrit and albumin is well reported in previous studies, this also strengthens the NLR as an inflammatory marker. Albumin is known to be inversely related to markers of chronic inflammatory states in patients with CKD and in maintenance hemodialysis (MHD) [32]. The stimulatory role of hypoalbuminemia on antidiuretic hormone release can lead to hyponatremia, which if over or aggressively corrected can lead to a non-inflammatory, osmotic, demyelinating injury in the basal pons of the brain stem [32]. The resulting hemodilution can play an initiating role in the occurrence of anemia-induced dilated cardiomyopathy (DCM) [33].

Limitations encountered included our inability to relate NLR with inflammatory mediators like tissue necrosis (TNF) and 1L-6.

\section{Conclusion}

The neutrophil lymphocyte ratio as a marker of pan systemic inflammation, is a useful tool for determining the presence and severity of inflammation in CKD. It was positively correlated with other inflammatory markers like uric acid, urine ACR and platelet lymphocyte ratio. The NLR was higher in the CKD cohorts and in females and, was positively related to the age, systolic blood pressure, uric acid, UACR and platelet lymphocyte ratio, as it was negatively related to the hematocrit, albumin and glomerular filtration rate. Aging, systolic hypertension, hyperuricemia, elevated urine ACR and declining kidney function independently predicted the NLR. The NLR could be a cheap, readily available tool for monitoring the progression of CKD in hospitals and community based settings. 


\section{Acknowledgements}

The authors appreciate the contributions of the staffs of the medical department and hematology unit for the support for the study.

\section{Conflicts of Interest}

The authors declare no conflicts of interest regarding the publication of this paper.

\section{References}

[1] Okyay, G.U., Inal, S., Oneç, K., et al. (2013) Neutrophil to Lymphocyte Ratio in Evaluation of Inflammation in Patients with Chronic Kidney Disease. Renal Failure, 35, 29-36. https://doi.org/10.3109/0886022X.2012.734429

[2] Manabe, I. (2011) Chronic Inflammation Links Cardiovascular, Metabolic and Renal Diseases. Circulation Journal, 75, 2739-2748. https://doi.org/10.1253/circj.CJ-11-1184

[3] Halazun, K.J., Aldoori, A., Malik, H.Z., et al. (2008) Elevated Preoperative Neutrophil to Lymphocyte Ratio Predicts Survival Following Hepatic Resection for Colorectal Liver Metastases. European Journal of Surgical Oncology, 34, 55-60. https://doi.org/10.1016/j.ejso.2007.02.014

[4] Guasti, L., Dentali, F., Castiglioni, L., et al. (2011) Neutrophils and Clinical Outcomes in Patients with Acute Coronary Syndromes and/or Cardiac Revascularisation. A Systematic Review on More than 34000 Subjects. Thrombosis and Haemostasis, 106, 591-599. https://doi.org/10.1160/TH11-02-0096

[5] Solak, Y., Yilmaz, M.I., Sonmez, A., et al. (2013) Neutrophil to Lymphocyte Ratio Independently Predicts Cardiovascular Events in Patients with Chronic Kidney Disease. Clinical and Experimental Nephrology, 17, 532-540.

https://doi.org/10.1007/s10157-012-0728-x

[6] Liu, J.H., Zhang, Y.J., Ma, Q.H., Sun, H.P., Xu, Y. and Pan, C.W. (2020) Elevated Blood Neutrophil to Lymphocyte Ratio in Older Adults with Cognitive Impairment. Archives of Gerontology and Geriatrics, 88, Article ID: 104041.

[7] Uduagbamen, P., Ogunkoya, J., AdebolaYusuf, A.O., et al. (2021) Hyperuricemia in Hypertension and Chronic Kidney Disease: Risk Factors, Prevalence and Clinical Correlates: A Descriptive Comparative Study. International Journal of Clinical Medicine, 12, 386-401. https://doi.org/10.4236/ijcm.2021.129035

[8] Tatar, E., Mirili, C., Isikyakar, T., et al. (2016) The Association of Neutrophil/Lymphocyte Ratio and Platelet/Lymphocyte Ratio with Clinical Outcomes in Geriatric Patients with Stage 3-5 Chronic Kidney Disease. Acta Clinica Belgica, 71, 221-226. https://doi.org/10.1080/17843286.2016.1159797

[9] Agabiti-Rosei, E. and Grassi, G. (2013) Beyond Gout: Uric Acid and Cardiovascular Diseases. Current Medical Research and Opinion, 29, 33-39. https://doi.org/10.1185/03007995.2013.790804

[10] Azab, B., Shah, N., Akerman, M. and McGinn, J.T. (2012) Value of Platelet/Lymphocyte Ratio as a Predictor of All-Cause Mortality after Non-ST Elevation Myocardial Infarction. Journal of Thrombosis and Thrombolysis, 34, 326-334. https://doi.org/10.1007/s11239-012-0718-6

[11] National Kidney Foundation (2012) KDOQI Clinical Practice Guideline for Diabetes and CKD: 2012 Update. American Journal of Kidney Diseases, 60, 850-886. https://doi.org/10.1053/j.ajkd.2012.07.005 
[12] Levey, A.S., Stevens, L.A., Schmid, C.H., et al. (2009) A New Equation to Estimate Glomerular Filtration Rate. Annals of Internal Medicine, 150, 604-612.

[13] Gerstein, H.C., Mann, J.F., Yi, Q., et al. (2001) Albuminuria and Risk of Cardiovascular Events, Death and Heart Failure in Diabetic and Non-Diabetic Individuals. JAMA, 286, 421-426. https://doi.org/10.1001/jama.286.4.421

[14] Meng, L., Yu, W., Wang, T., Zhang, L., Heerdt, P. and Gelb, A.W. (2018) Blood Pressure Targets in Perioperative Care. Provisional Considerations Based on a Comprehensive Literature Review. BMJ Hypertension, 72, 806-817.

[15] Lu, H., Luo, Y.Z., Wang, C. and Tu, H.T. (2016) The Urine Albumin-to-Creatinine Ratio Is a Reliable Indicator for Evaluating Complications of Chronic Kidney Disease and Progression in IgA Nephropathy in China. Clinics, 71, 243-250. https://doi.org/10.6061/clinics/2016(05)01

[16] Doualla, M., Halle, M.P., Moutchia, J., Tegang, S. and Ashuntantang, G. (2018) Determinants of Hyperuricemia in Non-Dialyzed Chronic Kidney Disease Patients in Three Hospitals in Cameroon. BMC Nephrology, 19, Article No. 169. https://doi.org/10.1186/s12882-018-0959-5

[17] Cappellini, M.D. and Mota, I. (2015) Anemia in Clinical Practice-Definition and Classification: Does Hemoglobin Change With Aging? Seminars in Hematology, 52, 261-269.

[18] Sevencan, N.U. and Ozkan, A.E. (2019) Associations between Neutrophil/Lymphocyte Ratio, Platelet/Lymphocyte Ratio, Albuminuria and Uric Acid and the Estimated Glomerular Filtration Rate in Hypertensive Patients with Chronic Kidney Disease Stages 1-3. Archives of Medical Science, 15, 1232-1239.

[19] Uduagbamen, P.K., Sanusi, M., Udom, O.B., et al. (2020) Preoperative Metabolic Acidosis in a Cardiovascular Surgical Intensive Care Unit: Risk factors, Clinical Correlates and Outcome. World Journal of Cardiovascular Surgery, 10, 226-241. https://doi.org/10.4236/wjcs.2020.1011025

[20] Hosmer, D.W. and Lameshow, S. (2000) Applied Logistic Regression. 2nd Edition, John Wiley \& Sons, New York. https://doi.org/10.1002/0471722146

[21] De Ciuceis, C., Amiri, F., Brassard, P., Endemann, D.H., Touyz, R.M. and Schiffrin, E.L. (2005) Reduced Vascular Remodeling, Endothelial Dysfunction, and Oxidative Stress in Resistance Arteries of Angiotensin II-Infused Macrophage Colony-Stimulating Factor-Deficient Mice: Evidence for a Role in Inflammation in AngiotensinInduced Vascular Injury. Arteriosclerosis, Thrombosis, and Vascular Biology, 25, 2106-2113.

[22] Furuncuoğlu, Y., Tulgar, S., Dogan, A.N., Cakar, S., Tulgar, Y.K. and Cakiroglu, B. (2016) How Obesity Affects the Neutrophil/Lymphocyte and Platelet/Lymphocyte Ratio, Systemic Immune-Inflammatory Index and Platelet Indices: A Retrospective Study. European Review for Medical and Pharmacological Sciences, 20, 1300-1306.

[23] Kocyigit, I., Eroglu, E., Unal, A., et al. (2013) Role of Neutrophil/Lymphocyte Ratio in Prediction of Disease Progression in Patients with Stage-4 Chronic Kidney Disease. Journal of Nephrology, 26, 358-365.

[24] Tamhane, U.U., Aneja, S., Montgomery, D., Rogers, E.K., Eagle, K.A. and Gurm, H.S. (2008) Association between Admission Neutrophil to Lymphocyte Ratio and Outcomes in Patients with Acute Coronary Syndrome. The American Journal of Cardiology, 102, 653-657. https://doi.org/10.1016/j.amjcard.2008.05.006

[25] Turak, O., Ozcan, F., Tok, D., et al. (2013) Serum Uric Acid, Inflammation and Non-Dipping Circadian Pattern in Essential Hypertension. The Journal of Clinical Hypertension, 15, 7-13. https://doi.org/10.1111/jch.12026 
[26] Uduagbamen, P.K., Oyelese, A.T., Alalade, B.A., Nwogbe, I.C. and Ofoh, C.J. (2021) Serum Sodium: Pattern, Determinants and Correlates in Nigerians with Hypertension and Chronic Kidney Disease. A Comparative Study. Clinical Nephrology and Research, 5, 1-8.

[27] Vaziri, N.D. (2014) Role of Dyslipidemia in Impairment of Energy Metabolism, Oxidative Stress, Inflammation and Cardiovascular Disease in Chronic Kidney Disease. Clinical and Experimental Nephrology, 18, 265-268.

https://doi.org/10.1007/s10157-013-0847-Z

[28] Dohi, Y. (2015) The First Step Aiming at the Prevention of Hypertension and Atherosclerosis. Identification of Individuals at High Risk of Hypertension. Rinsho Byori, 63, 1303-1309.

[29] Medina-Rosas, J., Gladman, D.D., Su, J. and Sabapathy, A. (2015) Utility of Untimed Single Urine Protein/Creatinine Ratio as a Substitute for 24-h Proteinuria for Assessment of Proteinuria in Systemic Lupus Erythematosus. Arthritis Research \& Therapy, 17, 296.

[30] Chen, C.F., Yang, W.C., Yang, C.Y., et al. (2015) Urinary Protein/Creatinine Ratio Weighted by Estimated Urinary Creatinine Improves the Accuracy of Predicting Daily Proteinuria. The American Journal of the Medical Sciences, 349, 477-487. https://doi.org/10.1097/MAJ.0000000000000488

[31] Xiang, L., Xiao, Y., Yong, W., et al. (2012) Association between Microalbuminuria and Subclinical Atherosclerosis Evaluated by Carotid Artery Intima-Media in Elderly Patients with Normal Renal Function. BMC Nephrology, 13, Article No. 37.

[32] Reijnders, T.D.Y., Janssen, W.M.T., Niamut, S.M.L., et al. (2020) Role of Risk Factors in Developing Osmotic Demyelination Syndrome during Correction of Hyponatremia: A Case Study. Cureus, 12, e6547. https://doi.org/10.7759/cureus.6547

[33] Efstratiadis, G., Konstantinou, D., Chytas, I. and Vergoulas, G. (2008) Cardio-Renal Anemia Syndrome. Hippokratia, 12, 11-16. 